\title{
Unusual Presentation of Focal Tubercular Meningoencephalitis in an Elderly Female
}

\author{
Ovais Khan ${ }^{1}$, Mohammed Irfan², Ganesh Lothe ${ }^{3}$, Jagtap Aniket ${ }^{4}$, Sourya Acharya ${ }^{5}$, Samarth Shukla ${ }^{6}$
}

${ }^{1}$ Datta Meghe Institute of Medical Sciences (Deemed to be University), Wardha, Maharashtra, India. ${ }^{2}$ Datta Meghe Institute of Medical Sciences (Deemed to be University), Wardha, Maharashtra, India. ${ }^{3}$ Datta Meghe Institute of Medical Sciences (Deemed to be University), Wardha, Maharashtra, India. ${ }^{4}$ Datta Meghe Institute of Medical Sciences (Deemed to be University), Wardha, Maharashtra, India. ${ }^{5}$ Department of Medicine, Datta Meghe Institute of Medical Sciences (Deemed to be University), Wardha, Maharashtra, India. ${ }^{6}$ Department of Pathology, Datta Meghe Institute of Medical Sciences (Deemed to be University), Wardha, Maharashtra, India.

\section{INTRODUCTION}

Central nervous system tuberculosis (CNS TB) can manifest as meningitis, tuberculoma, abscess, and encephalitis. Overall approximately $10 \%$ of all cases of tuberculosis have CNS involvement. We present a case of a 64-year-old female who presented to us with fever, abnormal behaviour in form of rage reactions and convulsions and was later diagnosed to be a case of focal tubercular meningoencephalitis. Tuberculosis (TB) is a global health problem. According to the World Health Organization (WHO) analysis in 2007, 9.27 million new cases of TB and 1.3 million TB-related deaths occur in immunocompetent subjects.[1] Central nervous system (CNS TB) is the most catastrophic extrapulmonary form of TB which is associated with a mortality rate of up to $60 \%$. In the survivors of CNS TB neurologic sequel may occur in up to $25 \%$ despite new neuroimaging diagnostic modalities and effective antituberculous therapy.[2] The incidence of tuberculosis varies from 9 cases per 100,000 population per year in the US to $110-165$ cases per 100,000 population in the developing countries in Asia and Africa.[3,4] The estimated mortality due to tuberculous meningitis in India is 1.5 per 100,000 population. ${ }^{[5]}$

\section{PRESENTATION OF CASE}

A 64-year-old female presented to us with chief complaints of fever and headache since 15 days, abnormal behaviour in form of rage reactions with abusive language, and 2 episodes of GTCS on the day of admission. The fever was intermittent and low grade. There was no history of diplopia, vomiting, unconsciousness, cough, expectoration, haemoptysis. The relatives being of village background presumed the rage reactions to be of paranormal origin and opted to go to some village priest, but when she developed seizures, they brought her to this hospital.

On examination GCS was E3-M5-V4. Patient was irritable, Pulse-120 bpm, RR 20 cycles per minute, BP-140/76 in right arm. CVS, RS and per abdomen examination was normal. CNS examination: Cranial nerves normal, she was moving all 4 limbs, Bilateral plantars showed withdrawal response. She did not cooperate for sensory examination but, she was withdrawing limbs from pinprick. Signs of meningeal irritation in form of neck stiffness, Kernig's sign and jolt accentuation test were positive.
Corresponding Author:

Dr. Sourya Acharya,

Department of Medicine,

DMIMS University, Sawangi (Meghe),

Wardha-442001, Maharashtra, India.

E-mail: souryaacharya74@gmail.com

DOI: $10.14260 /$ jemds/2019/769

Financial or Other Competing Interests: None.

How to Cite This Article:

Khan $O$, Irfan $M$, Lothe $G$, et al. Unusual presentation of focal tubercular meningoencephalitis in an elderly female. J. Evolution Med. Dent. Sci. 2019;8(47):3560 3562, DOI: 10.14260/jemds/2019/769

Submission 23-08-2019,

Peer Review 06-11-2019,

Acceptance 13-11-2019,

Published 25-11-2019. 
On investigation CBS, KFT, LFT, electrolytes, X-ray chest, fundus examination were all normal. MRI brain revealed abnormal leptomeningeal enhancement in the right high frontoparietal concavity with associated infarction and oedema, suggestive of focal meningoencephalitis (Figure 1).CSF examination was done which revealed: TLC $-220 / \mathrm{mm}^{3}$ with $80 \%$ lymphocytes. CSF glucose- $22 \mathrm{mg} / \mathrm{dl}$, proteins 250 $\mathrm{mg} / \mathrm{dl}$. CSF ADA - 48.6 IU (Strongly in favour of TB). CSF interferon gamma released assay (Quanti FERON-TB Gold test) was positive. CSF-PCR was negative for HSV.

A diagnosis of focal tuberculous meningoencephalitis was made and the patient was started with Intensive phase anti TB therapy (Isoniazid $300 \mathrm{mg}$ OD, Rifampicin $600 \mathrm{mg}$ OD, Pyrazinamide $750 \mathrm{mg}$ BID, Ethambutol $800 \mathrm{mg}$ OD), Injection Dexamethasone $4 \mathrm{mg}$ IV 8 hourly and. Inj levetiracetam 500 mg iV 12 hourly for seizures.

After 1 week of hospital stay the patient's general condition was improved fever subsided and the patient was discharged on oral Prednisolone $40 \mathrm{mg}$ along with ATT and Tab. Levetiracetam $500 \mathrm{mg}$ Bid. In the follow up after one month a repeat MRI of brain showed significant resolution of the lesion. (Figure 2)

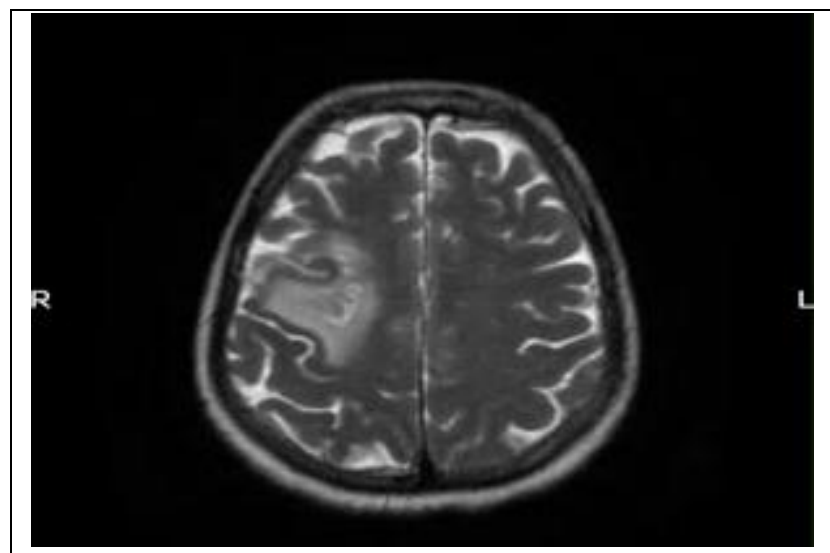

Figure 1. MRI Brain Showing Abnormal Leptomeningeal Enhancement in the Right High Frontoparietal Concavity with Associated Infarction and Oedema, Suggestive of Focal Meningoencephalitis

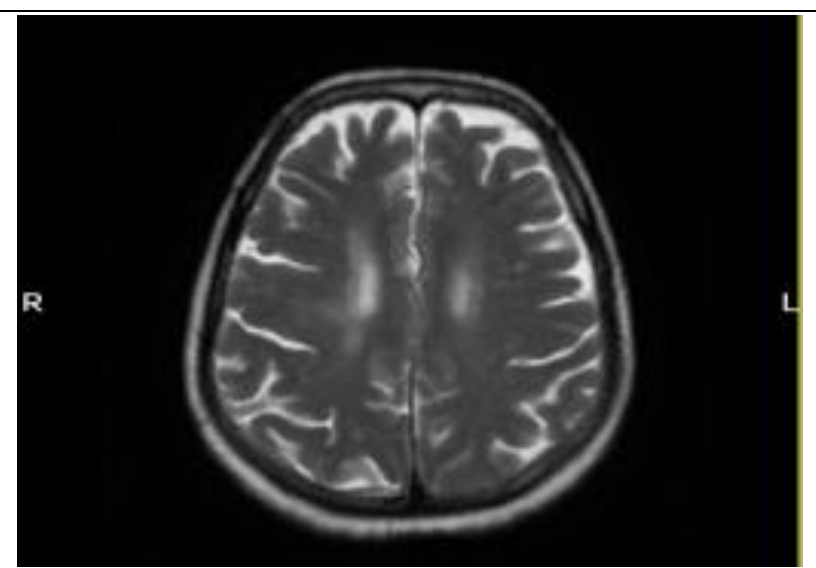

Figure 2. MRI Showing Resolution of the Lesion, 1 Month after ATT

\section{DISCUSSION}

Amongst the different variants of CNS TB, meningoencephalitis is the most severe, life-threatening form. It can occur in an acute or chronic variant. The predominant clinical presentations of TBM are headache, vomiting, fever and neck rigidity.[3] Our patient had these findings, along with that the patient's abnormal rage reactions, abusive aggression and seizures which pointed towards encephalitis which was later confirmed by neuroimaging and CSF analysis. Factors that are associated with poor outcome in TBM are extreme ages, stage of illness and development of hydrocephalus. ${ }^{[6-8]} \mathrm{A}$ study conducted by Hosoglu et al; [7] showed that drowsiness on admission is an important predictor for residual neurologic sequel.

The basic pathophysiology of CNS TB is the entry of the bacilli to CNS by hematogenous route from another focus. Rich and McCordock,[9] in their study suggested that CNS tuberculosis develops in two stages. The first stage heralds with formation of small tuberculous lesions known as "Rich's foci" in the CNS, either during the stage of bacteraemia of the primary tuberculous infection. The most favored sites in the brain are meninges, the subpial or subependymal surface of the brain or the spinal cord. These lesions may remain dormant for years after initial infection. In the second phase rupture or growth of one or more of these small tuberculous lesions produces various types of CNS tuberculosis. ${ }^{[9,10]}$ Rupture of Rich foci into the subarachnoid space or into the ventricular system results in meningitis.

Tuberculous encephalopathy, a syndrome is thought to be exclusively present in children. Diffuse cerebral oedema, convulsions, stupor or coma are characteristic of encephalitis. CSF is largely normal in pure encephalitis, but abnormal CSF picture suggests meningoencephalitis.[11]

As far as management of CNS TB is concerned; the first-line anti-TB agents recommended for the treatment include rifampicin (RIF), isoniazid (H), pyrazinamide (PZA), ethambutol (EMP) and/or streptomycin (S). Among the firstline drugs used in TBM, RIF, EMP and streptomycin have poor penetration across the blood-CSF barrier.[12] Currently, WHO recommends a 2-month treatment with 4 first-line drugs in the intensive phase, followed by a continuation phase with at least rifampicin and isoniazid for 4-10 months.[13] There is evidence that addition of corticosteroids improves both survival rate and neurological outcome in patients with tuberculous meningitis.[14-15]

\section{CONCLUSIONS}

Tubercular meningoencephalitis is the most catastrophic form of CNS TB. Classical clinical features, neuroimaging and recent diagnostic laboratory studies (CSF ADA, Quantiferon TB Gold assay) has improved the detection rate, so that early treatment can be initiated to limit the mortality and improve clinical outcomes in such cases.

\section{REFERENCES}

[1] World Health Organization. Global Tuberculosis Database. [Last Accessed on 2009 Dec]. http://www.who.int/research/en/

[2] Pehlivanoglu F, Yasar KK, Sengoz G. Prognostic factors of neurological sequel in adult patients with tuberculous meningitis. Neurosciences (Riyadh) 2010;15(4):262-7. 
[3] Snider DE Jr, Roper WL. The new tuberculosis. N Engl J Med 1992;326(10):703-5.

[4] Harries AD. Tuberculosis and human immunodeficiency virus infection in developing countries. Lancet 1990;335(8686):387-90.

[5] Chakraborty AK. Estimating mortality from tuberculous meningitis in a community: use of available epidemiological parameters in the Indian context. Ind J Tub 2000;47(1):9-13.

[6] Yasar KK, Pehlivanoglu F, Sengoz G. Predictors of death in tuberculous meningitis: a multivariate analysis by 160 cases. Int J Tuberc Lung Dis 2010;14(10):330-5.

[7] Hosoglu S, Geyik MF, Balik I, et al. Predictors of outcome in patients with tuberculous meningitis. Int J Tuberc Lung Dis 2002;6(1):64-70.

[8] Pienaar M, Andronikou S, Van Toorn R. MRI to demonstrate diagnostic features and complications of TBM not seen with CT. Childs Nerv Syst 2009;25(8):9417.
[9] Rich AR, McCordock HA. Pathogenesis of tubercular meningitis. Bull John Hopkins Hosp 1933;52:5-37.

[10] Berger JR. Tuberculous meningitis. Curr Opin Neurol 1994;7(3):191-200.

[11] Udani PM, Dastur DK. Tuberculous encephalopathy with and without meningitis clinical features and pathological correlations. J Neurol Sci 1970;10(6):541-61.

[12] Donald PR. Cerebrospinal fluid concentrations of antituberculosis agents in adults and children. Tuberculosis (Edinb) 2010;90(5):279-92.

[13] Dye C, Scheele S, Dolin P, et al. Consensus statement. Global burden of tuberculosis: estimated incidence, prevalence and mortality by country. WHO surveillance and monitoring project. JAMA 1999;282(7):677-86.

[14] Holdiness MR. Management of tuberculous meningitis. Drugs 1990;39:224-33.

[15] Kumarvelu S, Prasad K, Khosla A, et al. Randomized controlled trial of dexamethasone in tuberculous meningitis. Tubercle Lung Dis 1994;75(3):203-7. 